\title{
CEMENTOBLASTOMA BENIGNO: RELATO DE CASO
}

\section{BENIGN CEMENTOBLASTOMA: CASE REPORT}

Marcelo Júnior Zanda
Marcelo Lupion Poleti
Thais Maria Freire Fernandes
Renata Sathler
Eduardo Sant'Ana
Alberto Consolaro

\section{RESUMO}

O cementoblastoma benigno é um tumor raro originado do ectomesênquima odontogênico, caracterizado pela proliferação de tecido semelhante ao cemento, associado à raiz dentária. Atendendo à sua raridade, o objetivo deste trabalho é apresentar o relato de um caso clínico de uma jovem de 19 anos com diagnóstico de cementoblastoma benigno, ressaltando os achados clínico, radiográfico e microscópico da lesão.

\section{Palavras-chave: CEMENTO DENTÁRIO;}

\begin{abstract}
Doutor em Estomatologia pela Faculdade de Odontologia de Bauru da Universidade de São Paulo

Doutorando em Estomatologia pela Faculdade de Odontologia de Bauru da Universidade de São Paulo

Professora de Ortodontia da Universidade Norte do Paraná - UNOPAR
\end{abstract}

Doutora em Ortodontia pela Faculdade de Odontologia de Bauru da Universidade de São Paulo

Professor Associado do Departamento de Estomatologia da Faculdade de Odontologia de Bauru da Universidade de São Paulo

Professor Titular do Departamento de Estomatologia da Faculdade de Odontologia de Bauru da Universidade de São Paulo

MANDÍBULA; NEOPLASIAS.

\begin{abstract}
The Benign Cementoblastoma is a rare tumor that originates from the odontogenic ectomesenchyme and it is characterized by the proliferation of a tissue that is similar to cement and is associated with the dental root. Because of its rarity, the goal of this study is to present a clinical case report of a 19-year female old with benign cementoblastoma located in the mandible, highlighting the clinical, radiographic and microscopic findings.
\end{abstract}

Keywords: Dental CEMENTUM; mandible; NEOPLASMS. 


\section{INTRODUÇÃO}

O Cementoblastoma Benigno (CB) é uma neoplasia odontogênica relativamente rara dos maxilares e foi primeiramente descrita por Dewey ${ }^{1}$ em 1927. A lesão é considerada como a única verdadeira neoplasia de origem do cemento. ${ }^{2} \mathrm{O} \mathrm{CB}$ geralmente ocorre em pessoas jovens e representa menos de $1 \%$ a $6,2 \%$ de todos os tumores odontogênicos. ${ }^{2}$ Caracteriza-se o CB por estar ligado à raiz do dente envolvido $\mathrm{e}$, mais frequentemente, tende a ser associado com o primeiro molar e segundo pré-molar na mandíbula, estando raramente associado com dente impactado ou parcialmente irrompido. ${ }^{3}$ Sintomas podem estar totalmente ausentes, quando ocorrem, a dor e a expansão são frequentes..$^{3-5}$ Além disso, o CB tem potencial de crescimento ilimitado ${ }^{3-6}$ e o tratamento recomendado é a enucleação da massa tumoral com a extração do dente envolvido. ${ }^{2-6}$

Assim, o objetivo deste trabalho é apresentar o relato de um caso clínico de cementoblastoma benigno, ressaltando os achados clínico, radiográfico e microscópico da lesão.

\section{RELATO DO CASO CLÍNICO}

Paciente leucoderma, 19 anos de idade, foi encaminhada ao serviço de Estomatologia da Faculdade de Odontologia de Bauru (FOBUSP) para avaliação de uma massa radiopaca com halo radiolúcido, de período evolutivo incerto, envolvendo as raízes do dente 46 (Figura 1), descoberta em exame radiográfico inicial para tratamento ortodôntico.

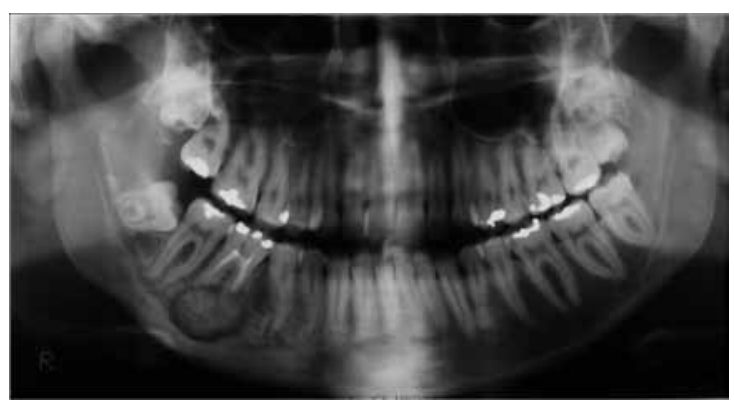

Figura 1 - EXAME RADIOGRÁfICO INICIAL

A história da doença atual revelou tratamento endodôntico do referido dente há seis meses, porém ainda com discreta sintomatologia dolorosa. Ao exame físico intraoral, observou-se expansão da cortical lingual na região do dente 46, confirmado pela radiografia oclusal total de mandíbula (Figura 2) que apresentou fenestração dessa parede.

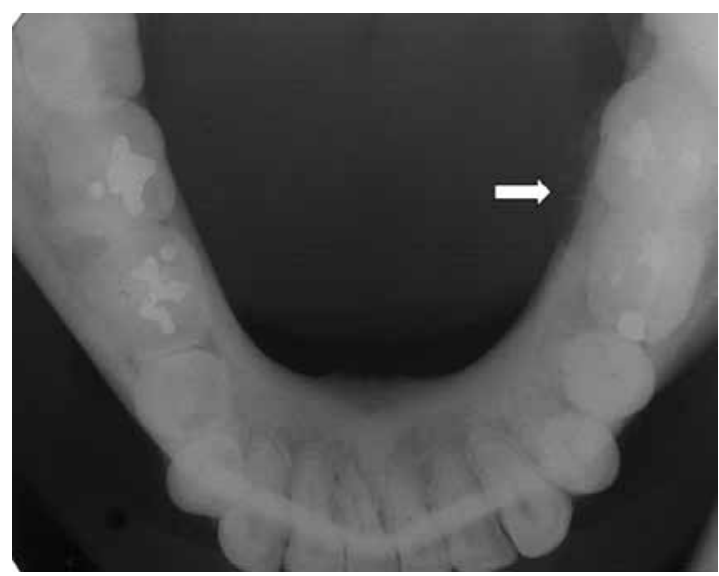

Figura 2 - RAdiografia oclusal tOtAl DE MANDÍBULA, OBSERVAR EXPANSÃO DA CORTICAL LINGUAL (SETA)

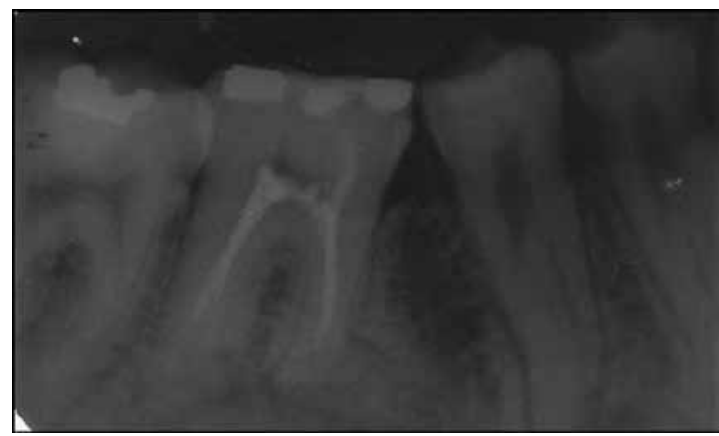

Figura 3 - RADIOGRAFIA PERIAPICAL INICIAL

As radiografias panorâmica (Figura 1) e periapical (Figura 3) demonstraram reabsorção radicular externa do dente envolvido, e baseado nos achados clínico e radiográfico o diagnóstico presuntivo foi de CB.

Foi realizado biópsia com broca trefina e a microscopia revelou massas irregulares de material mineralizado bem celularizado, ora cementoide ora osteoide, com lacunas dispostas irregularmente, linhas reversas basofílicas e muitas células gigantes multinucleadas, compatível com CB (Figura 4).

A opção terapêutica adotada foi a exodontia do dente 46 , enucleação da lesão e ostectomia periférica. Durante o procedimento cirúrgico, foi observada grande resistência aos movimentos de luxação devido à união raiz/lesão que culminou em fratura radicular. A lesão foi removida com as raízes dentárias (Figura 5) e confirmada microscopicamente pela conexão direta da lesão com a superfície. 


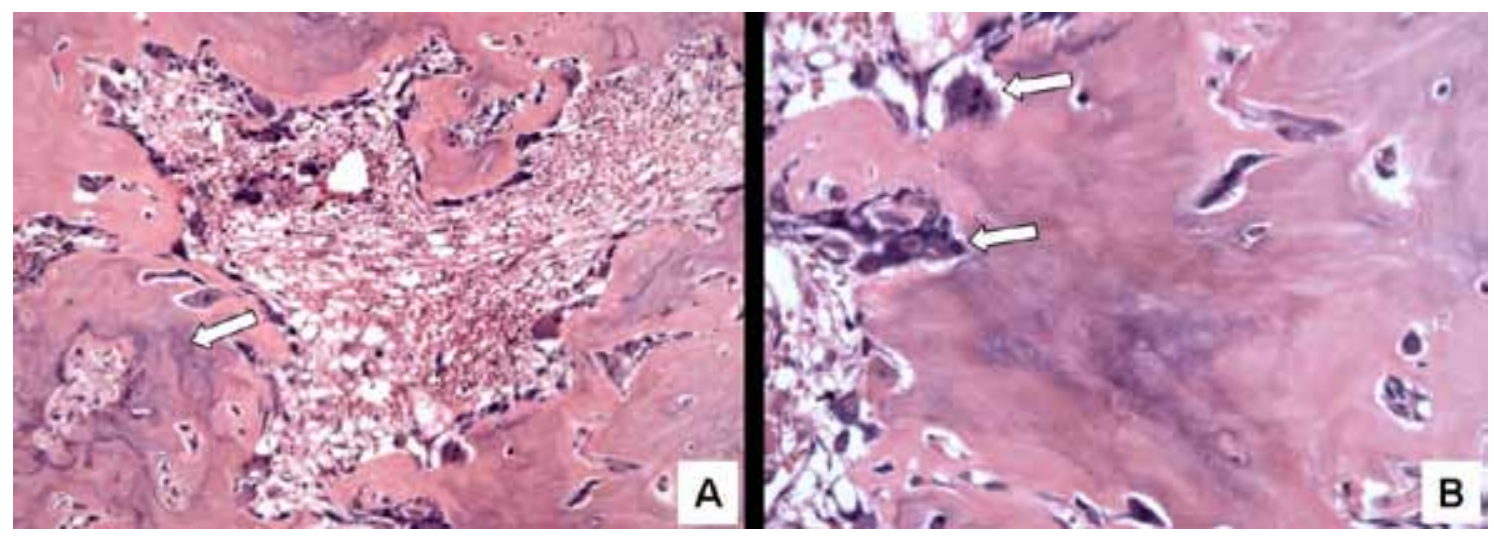

Figura 4 - AnÁlise histopatológica. Observar as linhas reVErsas basofílicas (A,SETA) E as CÉlulas GiGANTES MULTINUCLEADAS (B, SETAS)

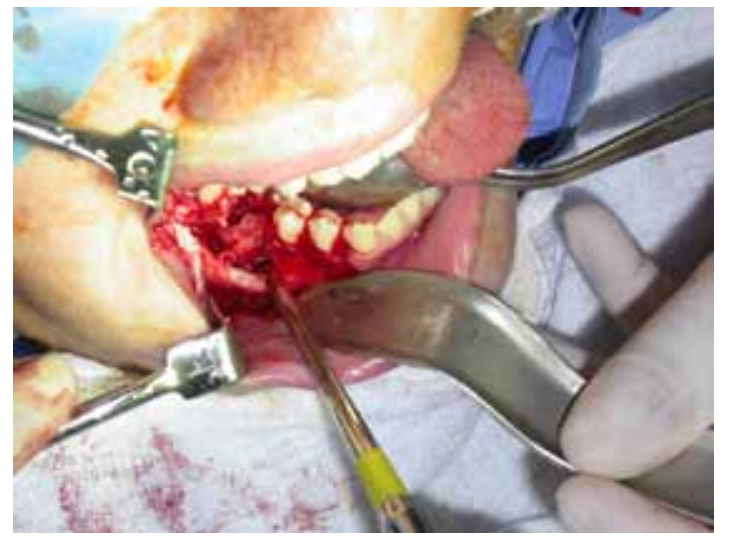

Figura 5 - Remoção da Lesão COM AS RAízeS DENTÁRIAS

Assim, baseado nesses achados, o diagnóstico final foi de CB. Atualmente a paciente se encontra em acompanhamento de três anos, sem sinais de recidiva da lesão (Figura 6).

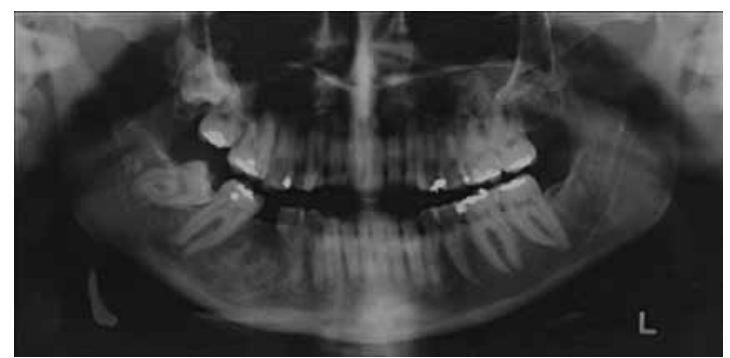

Figura 6 - CONTROLE RADIOGRÁFICO DE TRÊS ANOS

\section{DisCUSSÃo E CONCLUSÕES}

No passado, o $\mathrm{CB}$ foi reconhecido na classificação de tumores odontogênicos da Organização Mundial de Saúde como uma das neoplasias de cementoma. ${ }^{3}$ Recentemente, o CB é classificado como um tumor benigno constituído por ectomesênquima odontogênico com ou sem epitélio odontogênico. ${ }^{7}$ A lesão deriva do tecido ectomesenquimal, embora sua etiologia seja desconhecida. ${ }^{4,5}$

O CB é observado principalmente em jovens. Ulmansky et al. ${ }^{3}$ reviram a literatura e os autores relataram que cerca de $3 / 4$ dos pacientes (73\%) estão abaixo de 30 anos de idade. Embora tenha sido relatado que não há predileção significativa entre os dois gêneros, ${ }^{8,9}$ alguns autores ${ }^{10,11}$ indicam que os homens são afetados com mais frequência do que as mulheres. A mandíbula está envolvida com mais frequência do que a maxila ${ }^{2-6,8-11}$ e o dente mais afetado é o primeiro molar permanente, como encontrado no caso relatado. ${ }^{2}$ A lesão é de crescimento lento e geralmente assintomática, no entanto, dor e edema foram relatados em alguns casos. A expansão da cortical e assimetria facial são comuns. ${ }^{4-6,8,9}$

No caso relatado, o CB foi encontrado em uma mulher, jovem, localizado no primeiro molar permanente inferior direito, com sintomatologia, período evolutivo incerto e descoberto em exame radiográfico inicial para tratamento ortodôntico informações que estão de acordo com a literatura pesquisada.,3-6,8-11 Além disso, houve expansão da cortical lingual com fenestração dessa parede, como anteriormente descrito por Barbosa et al. ${ }^{12}$ $\mathrm{Na}$ história da doença atual, a paciente relatou endodontia prévia, que é comum nos casos de erro de diagnóstico desta lesão, confirmada pelas imagens radiográficas.

Radiograficamente, a lesão geralmente mostra uma massa radiopaca muitas vezes fusionada com uma ou mais raízes do dente envolvido e é limitada perifericamente por um halo radiolúcido. $\mathrm{O}$ caso relatado tem esta característica. Quando a relação íntima com as raízes está presente, a aparência radiográfica é quase patognomônica, ${ }^{3}$ isto porque 
a lesão poderia ser um osteoblastoma que cresceu e envolveu a raiz dentária.

Poucos autores têm relatado uma forma mais radiolúcida da lesão, o que eles consideram representar uma fase inicial não mineralizada da lesão. ${ }^{13}$

Microscopicamente, o cementoblastoma se assemelha ao osteoblastoma e osteoma osteoide, tendo como característica diferencial primária a fusão do tumor ao dente envolvido. A maior parte do tumor consiste em lençóis e trabéculas espessas de material mineralizado com lacunas dispostas irregularmente e linhas reversas basofílicas proeminentes. Tecido fibrovascular celular está presente entre as trabéculas mineralizadas. Células gigantes multinucleadas estão frequentemente presentes, e as trabéculas mineralizadas são geralmente revestidas por células semelhantes a blastos, características estas encontradas no caso relatado (Figura 4). A periferia de lesão, correspondente à área radiolúcida observada radiograficamente, é composta de matriz não mineralizada que geralmente é organizada em colunas radiantes. ${ }^{14}$

Há algumas lesões que devem ser distinguidas do $\mathrm{CB}$, dentre elas destacam-se o osteoblastoma e o osteoma osteoide. . $^{3-6,9,15} \mathrm{O}$ osteoblastoma é um tumor benigno do osso que se origina dos osteoblastos. A maioria dos osteoblastomas está entre 2 e $4 \mathrm{~cm}$, mas eles podem chegar a $10 \mathrm{~cm}$. Os achados clínicos, radiográficos e histopatológicos são muito semelhantes entre essa lesão e o CB, dependendo do estágio de evolução. Por causa dessas semelhanças, a diferença entre as duas lesões realmente depende de a lesão ser ou não unida ao dente e esta habilidade é provavelmente devida à histogênese final da célula tumoral responsável pela formação da lesão. ${ }^{14}$ Essa diferença pode ser observada cirurgicamente durante a exodontia do dente envolvido. No caso de CB, pode ocorrer fratura radicular em virtude do aumento da resistência nos movimentos de luxação devido à união raiz/lesão, e microscopicamente, pela conexão direta da lesão com a superfície radicular. ${ }^{16}$

O osteoma osteoide também é um tumor benigno do osso, porém é comum em ossos longos como fêmur e tíbia, sendo raro nos maxilares. São lesões pequenas, menos de $2 \mathrm{~cm}$, e, além disso, o tumor produz prostaglandinas que resultam em dor significativa aliviada por meio de inibidores das prostaglandinas, como a aspirina. Características essas que o diferem do CB e do osteoblastoma. ${ }^{14}$

Existem vários relatos de tratamento, desde o mais conservador; para tumores de reduzidas dimensões mediante tratamento endodôntico do dente afetado, apicectomia do mesmo e remoção do tumor, até mesmo a exodontia do dente envolvido e enucleação da lesão, esse o mais usual. A recorrência após qualquer um desses tratamentos é pouco comum e quando ocorre deve-se provavelmente à incompleta excisão da lesão. ${ }^{17}$ $\mathrm{Na}$ literatura, existem 13 casos de recorrência associados ao CB. A extração apenas do dente envolvido ou o dente com a remoção da lesão foi realizada em nove dos 13 casos recorrentes. Quatro casos foram tratados apenas com curetagem sem a extração do dente envolvido. ${ }^{8}$

Assim, o tratamento recomendado para o cementoblastoma deve consistir de remoção da lesão com o dente afetado, seguido de curetagem exaustiva ou ostectomia periférica. ${ }^{8}$ No caso relatado, foi optado por essa técnica, não havendo recorrência após três anos do procedimento cirúrgico.

\section{REFERÊNCIAS BIBLIOGRÁFICAS}

1. Dewey KW. Osteoma of a molar. Dent Cosmos 1927; 69:1143-9.

2. Lu Y, Xaun M., Takata T., Wang C., He Z., Zhou Z., et al. Odontogenic tumours. A demographic study of 759 cases in a Chinese population. Oral Surg Oral Med Oral Pathol Oral Radiol Endod 1998. 86:707-14.

3. Ulmansky M.,Hjorting-Hansen E., Praetorius F., Haque MF. Benign cementoblastoma; a review and five new cases. Oral Surg Oral Med Oral Pathol 1994. 77:48-55.

4. Piattelli A., Di Alberti L., Scarano A., Piattelli M. Benign cementoblastoma associated with an unerupted third molar. Oral Oncol 1998. 34:229-31.

5. Piatelli A., D'addona A., Piatelli M. Benign cemento-blastoma: Review of the literature and report of a case at unusual location. Acta Stomatol Belg 1990. 87:209-15.

6. Cundiff J. Developing cementoblastoma: Case report and update of differential diagnosis. Quintessence Int 2000. 31:191-5.

7. Barnes L., Eveson JW, Reichart P., Sidransky D. Pathology \& Genetics: Head and Neck Tumours. Geneva: WHO; 2005.

8. Brannon RB, Fowler CB, Carpenter WM, Corio RL. Cementoblastomas: an innocuous neoplasm? A clinicopathologic study of 44 cases and review of the literature with special emphasis on recurrence. Oral Surg Oral Med Oral Pathol Oral Radiol Endod 2002. 93:311-20. 
9. Regezi JA, Sciubba JJ. Oral pathology: Clinical-pathological correlations. Philadelphia: Saunders; 1989. p. 359-61.

10. Ohki K, Kumamoto H., Nitta Y., Nagasaka H., Kawamura H., Ooya K. Benign cementoblastoma involving multiple maxillary teeth: report of a case with a review of the literature. Oral Surg Oral Med Oral Pathol Oral Radiol Endod 2004. 97:53-8.

11. Monks FT, Bradley JC, Turner EP. Central osteoblastoma or cementoblastoma? A case report and 12-year review. Br J Oral Surg 1981. 19:29-37.

12. Barbosa NM, Cunha F., Leite M., Pinharanda H. Cementoblastoma da mandíbula - caso clínico. Rev Port Estomatol Cir Maxilofac 2004. 45:149-53.

13. Gingell JC, Lunin M., Beckerman T., Levy BA. Benign cementoblastoma. J. Oral Med 1984. $68: 8-11$.
14. Neville BW, Damm DD, Allen AM, Bouquot. Patologia oral \& maxillofacial. 2a. ed. Rio de Janeiro: Guanabara-Koogan; 2004. p. 545-8.

15. Junquera-Gutierrez L., Lopez-Arranz JS, Albertos-Castro JM, De Vicente-Rodriguez JC. Odontogenic tumors: classification, clinical features, diagnosis and treatment. Med Oral 1997. 2:94-101.

16. Fujita S., Takahashi H., Okabe H., Watanabe C., Sonobe H. A case of benign cementoblastoma. Oral Surg Oral Med Oral Pathol 1989. 68:64-8.

17. Biggs JT, Bennati FW. Surgicaly treating a benign cementoblastoma while retaining the envolving tooth. JADA 1995. 128:1.288-90.

Submetido em: 3/7/2012

Aceito em: 5/7/2012 